\title{
Patient-Reported Treatment Experience with Oral Rivaroxaban: Results from the Noninterventional XALIA Study of Deep-Vein Thrombosis
}

\author{
Stefan Cano ${ }^{1}$ Lorenzo Mantovani ${ }^{2}$ Kerstin Folkerts ${ }^{3}$ \\ Danja Monje $^{6}$ Jonas Schneider ${ }^{6}$ Martin van Eickels ${ }^{6}$ \\ Alexander G. G. Turpie ${ }^{10}$

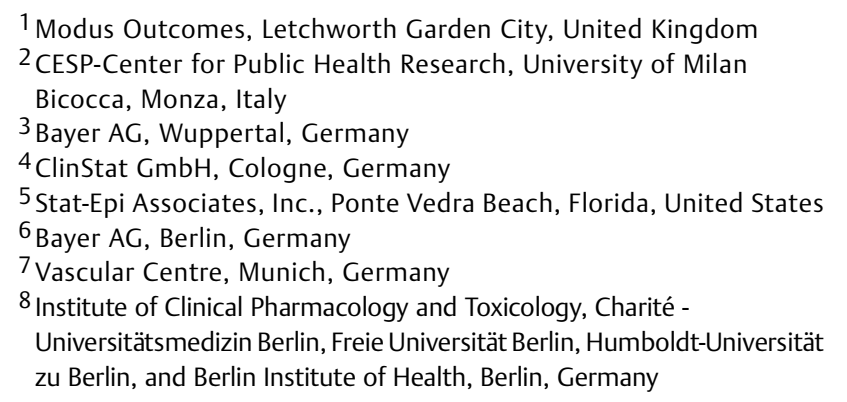

Martin Gebel ${ }^{3} \quad$ Kurtulus Sahin $^{4} \quad$ Elizabeth Zell ${ }^{5}$ Sylvia Haas ${ }^{7}$ Reinhold Kreutz ${ }^{8}$ Walter Ageno ${ }^{9}$

TH Open 2018;2:e139-e146.

\footnotetext{
${ }^{9}$ Department of Clinical and Experimental Medicine, University of Insubria, Varese, Italy

10 Department of Medicine, Hamilton Health Sciences, Hamilton, Ontario, Canada
}

\begin{abstract}
Address for correspondence Stefan Cano, PhD, Modus Outcomes, Suite 210b, Spirella Building, Letchworth Garden City SG6 4ET, United Kingdom (e-mail: Stefan.cano@modusoutcomes.com).
\end{abstract}

\begin{abstract}
Keywords

- ACTS

- anticoagulation

- patient experience

- rivaroxaban

- venous thromboembolism

For venous thromboembolism (VTE) treatment, patient satisfaction was shown to improve with rivaroxaban versus standard anticoagulation in the phase III EINSTEIN DVT and EINSTEIN PE trials. This substudy of the prospective, noninterventional XALIA study of rivaroxaban for deep-vein thrombosis treatment assessed if this was also observed in routine clinical practice. Patients enrolled in XALIA who received rivaroxaban or standard anticoagulation treatment were eligible for inclusion in this substudy. Treatment decisions were at the physician's discretion. Patients completed the 17item Anti-Clot Treatment Scale (ACTS, comprising a 12-item Burdens subscale, a 3-item Benefits subscale and one global item per subscale) during follow-up. The propensity score-matched set (PMS) was used for the main analysis; the adjusted safety analysis (ASAF) set was used for confirmatory purposes. Analyses by follow-up visit and subgroup, including age, sex, and previous VTE, were also conducted. The PMSACTS analysis included 458 rivaroxaban-treated and 434 standard anticoagulationtreated patients. Baseline demographic and clinical characteristics were generally similar across treatment arms. ACTS Burdens scores significantly improved with rivaroxaban versus standard anticoagulation (least-squares mean difference of $2.4 \pm 0.4$ points; $p<0.0001$ ); ACTS Benefits scores were numerically higher with rivaroxaban (least-squares mean difference of $0.2 \pm 0.1$ points; $p=0.2$ ). Similar findings occurred across follow-up visits and subgroups. Results were confirmed in the ASAF-ACTS analysis. Consistent with phase III analyses, rivaroxaban was associated with improved ACTS Burdens scores; ACTS Benefits scores numerically favored rivaroxaban, although without reaching statistical significance.
\end{abstract}

received

September 29, 2017

accepted

February 19, 2018
DOI https://doi.org/

10.1055/s-0038-1641679. ISSN 2512-9465. (c) 2018 Georg Thieme Verlag KG Stuttgart · New York
License terms

(c) (i) 


\section{Introduction}

Anticoagulant treatment for venous thromboembolism (VTE), comprising deep-vein thrombosis (DVT) and pulmonary embolism (PE), is associated with various benefits and burdens, some of which are therapy specific. Traditional therapies have several well-known limitations; agents such as lowmolecular-weight heparin (LMWH) are administered via injection, which makes self-administration problematic for some patients. ${ }^{1}$ Many patients also have a dislike or fear of needles, ${ }^{2}$ meaning that repeated injections may not be suitable for them. Vitamin $\mathrm{K}$ antagonists (VKAs) require regular anticoagulation monitoring, which contributes to making their use in the outpatient setting suboptimal. A meta-analysis of randomized trials and cohort studies showed that in the first month of treatment, patients were within the target international normalized ratio (INR) range of 2.0 to 3.0 only $56 \%$ of the time. ${ }^{3}$ Even when the first 3 months of treatment were excluded from the meta-analysis, the time in therapeutic range was only around $75 \%$. The interaction of VKAs with foods containing high levels of vitamin $\mathrm{K}$, other medications, and alcohol contributes to the need for monitoring and management of adverse effects, making their use more burdensome. ${ }^{4}$ VKAs have slow and variable onsets of action, and there is considerable variation between individuals in terms of their response to therapy. Other concerns associated with anticoagulation treatment may adversely affect patients' attitudes toward therapy, for example, the perceived risk of bleeding events.

In addition to treatment efficacy, the issues described earlier can impact the patient's subjective impression of the treatment process. Employing measures to assess and quantify parameters such as treatment satisfaction or enhancement of the patient experience is becoming common practice as part of clinical studies, especially as treatment satisfaction has a positive association with adherence and persistence. ${ }^{5,6}$ The non-VKA oral anticoagulants (apixaban, dabigatran, edoxaban, and rivaroxaban) can potentially reduce many of the burdens associated with standard therapies. For example, their oral route of administration, combined with a lack of requirement for routine anticoagulation monitoring, would be expected to reduce the burdens associated with repeated injections and INR testing.

The phase III EINSTEIN DVT and EINSTEIN PE studies demonstrated a higher degree of patient treatment satisfaction with rivaroxaban versus standard anticoagulation..$^{7-10}$ The noninterventional XALIA study of rivaroxaban versus standard anticoagulation for the treatment of DVT in routine clinical practice was conducted subsequently. One of the aims of XALIA was to determine whether improved patient experience with rivaroxaban was replicated outside the clinical trial setting $;{ }^{11}$ this analysis presents the results of these assessments.

\section{Materials and Methods}

The XALIA study methods are described in the XALIA primary article. ${ }^{11}$ The methodology for the Anti-Clot Treatment Scale (ACTS) substudy is presented below.

\section{Patients}

All patients enrolled in XALIA who received rivaroxaban or standard anticoagulation treatment were eligible for inclusion in this substudy. Patients were aged $\geq 18$ years, with objectively confirmed DVT (after approval of rivaroxaban in the PE indication, patients with DVT and concomitant PE were also eligible) and an indication for at least 3 months of anticoagulation treatment. The type, dose, and duration of anticoagulant drug therapy administered to each patient were at the discretion of the attending physician. Patients who received rivaroxaban alone, or who initially received heparin/fondaparinux for a maximum of 48 hours before enrolment, were included in the rivaroxaban cohort, consistent with the approach in the EINSTEIN DVT phase III trial. ${ }^{7}$ Patients who received initial heparin or fondaparinux for $>2$ to 14 days with or without a VKA for 1 to 14 days before switching to rivaroxaban were designated as "early switchers" and were not included in the analysis presented here.

\section{Anti-Clot Treatment Scale}

Patients completed the anticoagulation-specific ACTS during follow-up visits; country-specific and linguistically validated versions of the questionnaire were used. The ACTS comprises 17 items representing negative and positive aspects of anticoagulation treatment: ACTS Burdens (12 scale items plus 1 global item regarding burdens); ACTS Benefits ( 3 scale items plus 1 global item regarding benefits). Item scores were summed across domains to give an ACTS Burdens score ranging from 12 to 60 and an ACTS Benefits score ranging from 3 to 15, with higher scores in both indicating a more positive patient experience with anticoagulation treatment; scores for global items were not included in the calculations. The ACTS was determined at days 1 to 44 (Visit 1 ), days 45 to 134 (Visit 2), days 135 to 224 (Visit 3), days 225 to 314 (Visit 4), and any time from day 315 onward (Visit 5). The questions comprising the ACTS questionnaire are shown in the -Supplementary Material (-Table S1).

\section{Propensity Score-Adjusted Analysis}

Propensity score adjustment was used to address the imbalances in baseline characteristics of the two treatment groups in the safety analysis set. Briefly, analyses were conducted in two ways, in a set of matched pairs as well as stratified by eight homogeneous subclasses, both sets derived with propensity scores. Propensity scores, matched pairs, and subclasses were developed by an independent statistician without knowledge of outcome events and addressed allocation bias via adjustment of the potential effect of unbalanced covariates. ${ }^{12,13}$ Matched pairs based on the propensity scores were created using the greedy algorithm. ${ }^{14}$ The propensity score derivation and stratified analysis methodologies are included in the Supplementary Material of the XALIA primary article. ${ }^{11}$ The main analysis of the ACTS results in this study was conducted on the set of patients with matched propensity scores (the propensity score-matched set [PMS]) who had at least one nonmissing ACTS assessment after implementation of the imputation method (termed the PMS- 
ACTS set). As a consequence, only a subset of patients consisted of matched pairs. Alternatively, patients with propensity scores that were too high/low were removed from the safety set and eight homogenous subclasses were created for the adjusted safety analysis (ASAF) set. A sensitivity analysis was conducted in the adjusted safety set of patients who had at least one nonmissing ACTS assessment after implementation of the imputation method (ASAFACTS).

\section{Subgroups}

Several subgroups were also assessed for differences in ACTS Burdens and Benefits scores between treatments. These subgroups were as follows: age $(<60, \geq 60$ years); body mass index $\left(\leq 25,>25\right.$ to $\left.\leq 35,>35 \mathrm{~kg} / \mathrm{m}^{2}\right)$; sex (male, female); hospitalization for index events (yes, no); immobilization (yes, no); language (Dutch, German, French, English, Italian, Swedish, Spanish, Canadian English); provoked VTE (yes, no); reason for treatment (patient's age, patient's living conditions, comorbidities, distance to treating physician, medical or hospital guidelines, availability of drug, price of drug, type of health insurance, other); renal disease (yes, no); active cancer at baseline (yes, no); active cancer at baseline (excluding patients on VKA; yes, no); race (white, black, Asian, not reported); region (western Europe, Canada and Israel, eastern Europe); first available weight ( $\leq 70,>70$ to $<90, \geq 90 \mathrm{~kg}$ ); country (France, Germany, Spain, rest); chronic heart failure (yes, no); diabetes (yes, no); first available creatinine clearance $(<30, \geq 30$ to $<50, \geq 50$ to $<80$, $\geq 80 \mathrm{~mL} / \mathrm{min}$ ); patient health insurance (public, fully private, partially private, other [including missing]); cardiovascular disease (yes, no); stroke (yes, no); previous major bleeding event (yes, no); previous VTE (yes, no); thrombophilia (yes, no); venous insufficiency (yes, no); history of hypertension (yes, no); and chronic obstructive pulmonary disease (yes, no).

\section{Statistical Analysis}

The ACTS was completed in accordance with the developer's guidelines. ${ }^{15}$ In instances for which more than $50 \%$ of questions were missing responses, the scale was considered to be incomplete; partial completion where missing responses were below $50 \%$ was addressed by scale-specific mean imputation.

A mixed-model repeated-measures analysis using an unstructured covariance matrix was used to analyze the questionnaire data because the questionnaire responses were multiple measurements on patient experience with treatment over a period of time. The mixed model included ACTS as outcome, and treatment, cancer at baseline, and treatment by visit interaction as covariates. From this model, least square (LS) means for each treatment group and treatment differences and corresponding standard errors (SEs) were presented per visit. The same analysis without treatment by visit interaction for the overall effect with LS means and SEs is shown. For ASAF-ACTS, the LS means were calculated individually per strata and treatment and then combined with a stratified-based combining rule. All statis- tical significance testing was performed at a two-sided $0.05 \alpha$ level, and all data analysis was done using SAS statistical software package version 9.4 (SAS Institute, Cary, North Carolina, United States).

\section{Results}

\section{Patients and ACTS Completion Rates}

Of the 5,136 patients who received study medication in XALIA, 4,768 (92.8\%) were included in the safety analysis set (368 [7.2\%] early switchers were excluded from the primary analysis). Subsequently, 253 patients were excluded from the ASAF because they had propensity scores that were either too high or too low. This left an ASAF set of 4,515 patients, 2,505 (55.5\%) of who received rivaroxaban and 2,010 (44.5\%) who received standard anticoagulation. A total of 1,124 matched pairs (2,248 patients) were included in the PMS set.

Because several patients in XALIA had missing ACTS data, the PMS-ACTS set comprised 892 patients in total, of which 458 (51.3\%) received rivaroxaban and 434 (48.7\%) received standard anticoagulation. The ASAF-ACTS set included 1,726 patients $(1,007$ [58.3\%] for rivaroxaban, 719 [41.7\%] for standard anticoagulation).

\section{Baseline Demographics and Clinical Characteristics}

The baseline demographics and clinical characteristics of the PMS-ACTS set are shown in - Table 1. Patients in the rivaroxaban cohort were generally younger, had higher rates of unprovoked VTE, and had lower rates of renal impairment, concomitant PE, and active cancer than those in the standard anticoagulation group. The baseline characteristics of both analysis sets were similar to those of the full safety analysis set. $^{11}$

For the PMS-ACTS set, the countries with the highest rates of representation were France (30.6\%) and Germany (27.2\%). A similar pattern was seen with the ASAF-ACTS set, with France and Germany again forming the largest geographical cohorts (30.9 and $29.5 \%$, respectively).

\section{ACTS Burdens}

\section{PMS-ACTS Analysis Set}

The overall ACTS Burdens score (LS mean difference) was $2.4 \pm 0.4$ points higher (higher scores indicating a reduced burden) in the PMS-ACTS set in the rivaroxaban group versus the standard anticoagulation group (LS mean scores of 56.1 and 53.7, respectively; $p<0.0001$ ), indicative of a significantly reduced burden with rivaroxaban. The higher scores with rivaroxaban were consistent over time, with the LS mean difference in scores ranging from 2.2 (Visits 2 and 4 ) to 2.9 (Visit 3); the differences were significant at each visit (-Table 2).

\section{ASAF-ACTS Analysis Set}

The overall ACTS Burdens score (LS mean difference) was $2.4 \pm 0.4$ points higher in the ASAF-ACTS set in the rivaroxaban group versus the standard anticoagulation group (LS 
Table 1 Baseline demographics and clinical characteristics of patients in the XALIA treatment satisfaction substudy (PMSACTS set)

\begin{tabular}{|c|c|c|}
\hline Characteristic $^{\mathrm{a}}$ & $\begin{array}{l}\text { Rivaroxaban } \\
(N=458)\end{array}$ & $\begin{array}{l}\text { Standard anti- } \\
\text { coagulation }^{b} \\
(N=434)\end{array}$ \\
\hline Mean age, y (SD) & $60.1(15.7)$ & $61.7(16.6)$ \\
\hline \multicolumn{3}{|l|}{ Age category } \\
\hline$<60$ y & $198(43.2)$ & $191(44.0)$ \\
\hline$\geq 60 y$ & $260(56.8)$ & $243(56.0)$ \\
\hline Male sex & $196(42.8)$ & $206(47.5)$ \\
\hline \multicolumn{3}{|l|}{ Weight } \\
\hline$<50 \mathrm{~kg}$ & $4(0.9)$ & $6(1.4)$ \\
\hline$\geq 50$ to $70 \mathrm{~kg}$ & $97(21.2)$ & 95 (21.9) \\
\hline$>70$ to $<90 \mathrm{~kg}$ & $157(34.3)$ & $150(34.6)$ \\
\hline$\geq 90 \mathrm{~kg}$ & $119(26.0)$ & $111(25.6)$ \\
\hline Missing & $81(17.7)$ & $72(16.6)$ \\
\hline \multicolumn{3}{|c|}{ First available creatinine clearance } \\
\hline$<30 \mathrm{~mL} / \mathrm{min}$ & $1(0.2)$ & $8(1.8)$ \\
\hline $\begin{array}{l}\geq 30 \text { to }<50 \\
\mathrm{~mL} / \mathrm{min}\end{array}$ & $20(4.4)$ & $21(4.8)$ \\
\hline $\begin{array}{l}\geq 50 \text { to }<80 \\
\mathrm{~mL} / \mathrm{min}\end{array}$ & $95(20.7)$ & $77(17.7)$ \\
\hline$\geq 80 \mathrm{~mL} / \mathrm{min}$ & 201 (43.9) & $160(36.9)$ \\
\hline Missing & $141(30.8)$ & $168(38.7)$ \\
\hline \multicolumn{3}{|l|}{ Index diagnosis } \\
\hline DVT only & 409 (89.3) & $382(88.0)$ \\
\hline DVT with PE & $49(10.7)$ & $52(12.0)$ \\
\hline \multicolumn{3}{|l|}{ Type of VTE } \\
\hline Provoked & $143(31.2)$ & $155(35.7)$ \\
\hline Unprovoked & $315(68.8)$ & $279(64.3)$ \\
\hline Previous VTE & $124(27.1)$ & $109(25.1)$ \\
\hline $\begin{array}{l}\text { Active cancer at } \\
\text { baseline }\end{array}$ & $40(8.7)$ & $46(10.6)$ \\
\hline $\begin{array}{l}\text { Known thrombo- } \\
\text { philic condition }\end{array}$ & $35(7.6)$ & $35(8.1)$ \\
\hline $\begin{array}{l}\text { Previous major } \\
\text { bleeding episode }\end{array}$ & $11(2.4)$ & $8(1.8)$ \\
\hline
\end{tabular}

Abbreviations: ACTS, Anti-Clot Treatment Scale; DVT, deep-vein thrombosis; PE, pulmonary embolism; PMS, propensity score matched set; SD, standard deviation; VTE, venous thromboembolism.

${ }^{a} n(\%)$ unless otherwise stated.

bStandard anticoagulation consisted of initial treatment with unfractionated heparin, low-molecular-weight heparin, or fondaparinux, which could overlap with and be followed by an oral vitamin $\mathrm{K}$ antagonist.

${ }^{\text {C}}$ Cancer was not considered when defining DVT as provoked or unprovoked.

mean scores of 55.2 and 52.9, respectively; $p=0.0001$ ), indicative of a significantly reduced burden with rivaroxaban. The higher scores with rivaroxaban were consistent over time, with LS mean difference in scores ranging from 2.0
(Visit 2) to 2.8 (Visit 3); the differences were significantly different at each visit ( - Table 3 ).

\section{Subgroups}

Almost all patient subgroups in the PMS-ACTS and ASAFACTS sets showed statistically significant differences in ACTS Burdens scores between treatment groups in favor of rivaroxaban, both for overall scores and by treatment visit. Treatment comparisons for selected subgroups are shown in - Fig. 1.

\section{ACTS Benefits}

\section{PMS-ACTS Analysis Set}

The overall ACTS Benefits score (LS mean difference) was $0.2 \pm 0.2$ points higher in the PMS-ACTS set in the rivaroxaban group versus the standard anticoagulation group, although this difference was not significant (LS mean scores of 12.1 and 11.9 , respectively; $p=0.2$ ). The similar ACTS Benefits scores between the two treatment groups were consistent over time, with the LS mean difference in scores ranging from -0.1 (Visit 1 ) to 0.5 (Visit 2; - Table 4).

\section{ASAF-ACTS Analysis Set}

The overall ACTS Benefits score (LS mean difference) was $0.2 \pm 0.1$ points higher in the ASAF-ACTS set in the rivaroxaban group versus the standard anticoagulation group, although this difference was not statistically significant (LS mean scores of 11.9 and 11.8 , respectively; $p=0.4$ ). The similar ACTS Benefits scores between the two treatment groups were consistent over time, with the LS mean difference in scores ranging from $<0.1$ (Visit 5) to 0.2 (Visits 1,3 , and 4 ; - Table 5 ).

\section{Subgroups}

As with the total PMS-ACTS and ASAF-ACTS results, the majority of subgroups showed no statistically significant differences between treatment groups for ACTS Benefits scores, either for the overall scores or by treatment visit. For those subgroups that did show differences, almost all were at Visit 2. Treatment comparisons for selected subgroups are shown in -Fig. 2.

\section{Discussion}

The XALIA ACTS substudy compared treatment experience with DVT (also DVT plus concomitant PE after the approval of rivaroxaban in the PE indication) in patients who were treated with rivaroxaban or standard anticoagulation. The results demonstrated that patients given rivaroxaban had an improved treatment experience, both overall and across study time points, in terms of finding the treatment less burdensome than standard anticoagulation. Similarly, the ACTS Benefits scores showed a numerical trend in favor of rivaroxaban. These findings for the ACTS Burdens scores are consistent with results from the ACTS substudy of the EINSTEIN DVT trial. ${ }^{9}$ The PMS-ACTS and ASAF-ACTS analysis sets were similar to the overall XALIA safety analysis set in 
Table 2 ACTS Burdens scores by visit (PMS-ACTS set)

\begin{tabular}{|l|l|l|l|l|}
\hline Visit & $\begin{array}{l}\text { Rivaroxaban/standard } \\
\text { anticoagulation } \\
\text { patients }(\boldsymbol{n} / \boldsymbol{n})^{\mathrm{a}}\end{array}$ & $\begin{array}{l}\text { Rivaroxaban/standard } \\
\text { anticoagulation } \\
\text { scores (LS mean/LS } \\
\text { mean) }\end{array}$ & $\begin{array}{l}\text { LS mean difference } \\
\text { (SE) }\end{array}$ & $p$-Value \\
\hline Overall & $458 / 434$ & $56.1 / 53.7$ & $2.4(0.4)$ & $<0.0001$ \\
\hline 1 & $333 / 295$ & $55.2 / 52.5$ & $2.7(0.5)$ & $<0.0001$ \\
\hline 2 & $307 / 279$ & $56.0 / 53.8$ & $2.2(0.5)$ & $<0.0001$ \\
\hline 3 & $179 / 161$ & $56.1 / 53.2$ & $2.9(0.6)$ & $<0.0001$ \\
\hline 4 & $68 / 75$ & $56.7 / 54.5$ & $2.2(0.7)$ & 0.002 \\
\hline 5 & $69 / 77$ & $56.5 / 54.1$ & $2.4(0.6)$ & 0.0002 \\
\hline
\end{tabular}

Abbreviations: ACTS, Anti-Clot Treatment Scale; LS, least squares; PMS, propensity score matched set; SE, standard error.

${ }^{a}$ ACTS Burdens scores had no effect on treatment discontinuation in the PMS-ACTS analysis set. Patients treated with standard anticoagulation treatment discontinued 1.6 times more than patients treated with rivaroxaban.

respect of baseline demographic and clinical characteristics. The exceptions to this were that patients in the PMS-ACTS set were on average $\sim 1$ year older (60.9 vs. 59.8 years) and had higher rates of previous VTE (26.1 vs. $23.3 \%)$ than patients in the safety analysis set.

The completion rates of the ACTS for the rivaroxaban and standard anticoagulation groups in the ACTS substudy in XALIA were consistent with that observed for EINSTEIN DVT, where around $40 \%$ of all patients were analyzed in the treatment satisfaction substudy. Similar to EINSTEIN DVT, the open-label nature of treatment administration in XALIA meant that patients were able to evaluate the impact of the therapy directly because they were not given dummy controls or subject to sham INR monitoring. In addition, the realworld nature of the XALIA study also means that the findings are reflective of actual clinical practice. Subgroup analysis by baseline patient demographics and clinical characteristics revealed a similar pattern to the main analyses for ACTS Burdens and Benefits scores. For the ACTS Burdens scores, most subgroups showed significant between-treatment differences favoring rivaroxaban for overall scores and scores by visit. Of note, the effect size was smaller or showed a marginal trend toward standard anticoagulation in patients with provoked VTE; a more robust follow-up and health service contact may explain the almost equivalent response to rivaroxaban. In addition, the ACTS Burdens scores did not show a statistically significant between-treatment difference in patients with thrombophilia; again, a more intensive follow-up may explain this finding. For ACTS Benefits scores, there was a numerical trend favoring rivaroxaban across visits and subgroups, although only a limited number of subgroups showed statistically significant differences, and these were almost all at Visit 2.

A limitation of this study is that it assessed only patient experience over a relatively short period of time (the median treatment duration in XALIA was 181 days with rivaroxaban and 190 days with standard anticoagulation); therefore, it would also be of interest to determine whether the patient experience findings observed in XALIA persist in the longer term. It is important to note that the ACTS questionnaire is a specific measure that focuses on all medical aspects of the treatment experience that are important to patients and gathers information on all aspects of anticoagulation therapy, both positive and negative. ${ }^{9}$ Therefore, a widely used, generic measure (e.g., the Treatment Satisfaction Questionnaire for Medication version II [TSQM II]) focusing on the medication per se could complement the ACTS results. ${ }^{9,16}$ It is often highlighted that the use of two measures offers

Table 3 ACTS Burdens scores by visit (ASAF-ACTS set)

\begin{tabular}{|l|l|l|l|l|}
\hline Visit & $\begin{array}{l}\text { Rivaroxaban/standard } \\
\text { anticoagulation } \\
\text { patients }(\boldsymbol{n} / \boldsymbol{n})\end{array}$ & $\begin{array}{l}\text { Rivaroxaban/standard } \\
\text { anticoagulation } \\
\text { scores (LS mean/LS } \\
\text { mean) }\end{array}$ & $\begin{array}{l}\text { LS mean difference } \\
\text { (SE) }\end{array}$ & $p$-Value \\
\hline Overall & $1,007 / 718$ & $55.2 / 52.9$ & $2.4(0.4)$ & 0.0001 \\
\hline 1 & $743 / 475$ & $54.6 / 52.3$ & $2.5(0.5)$ & 0.0003 \\
\hline 2 & $656 / 450$ & $55.1 / 53.2$ & $2.0(0.5)$ & 0.001 \\
\hline 3 & $371 / 246$ & $55.2 / 52.9$ & $2.8(0.5)$ & 0.0004 \\
\hline 4 & $166 / 120$ & $55.6 / 53.5$ & $2.7(0.8)$ & 0.009 \\
\hline 5 & $155 / 115$ & $55.4 / 52.8$ & $2.7(0.8)$ & 0.002 \\
\hline
\end{tabular}

Abbreviations: ACTS, Anti-Clot Treatment Scale; ASAF, adjusted safety analysis; LS, least squares; SE, standard error. 
e144 Patient Experience with Rivaroxaban Treatment in the XALIA Study Cano et al.

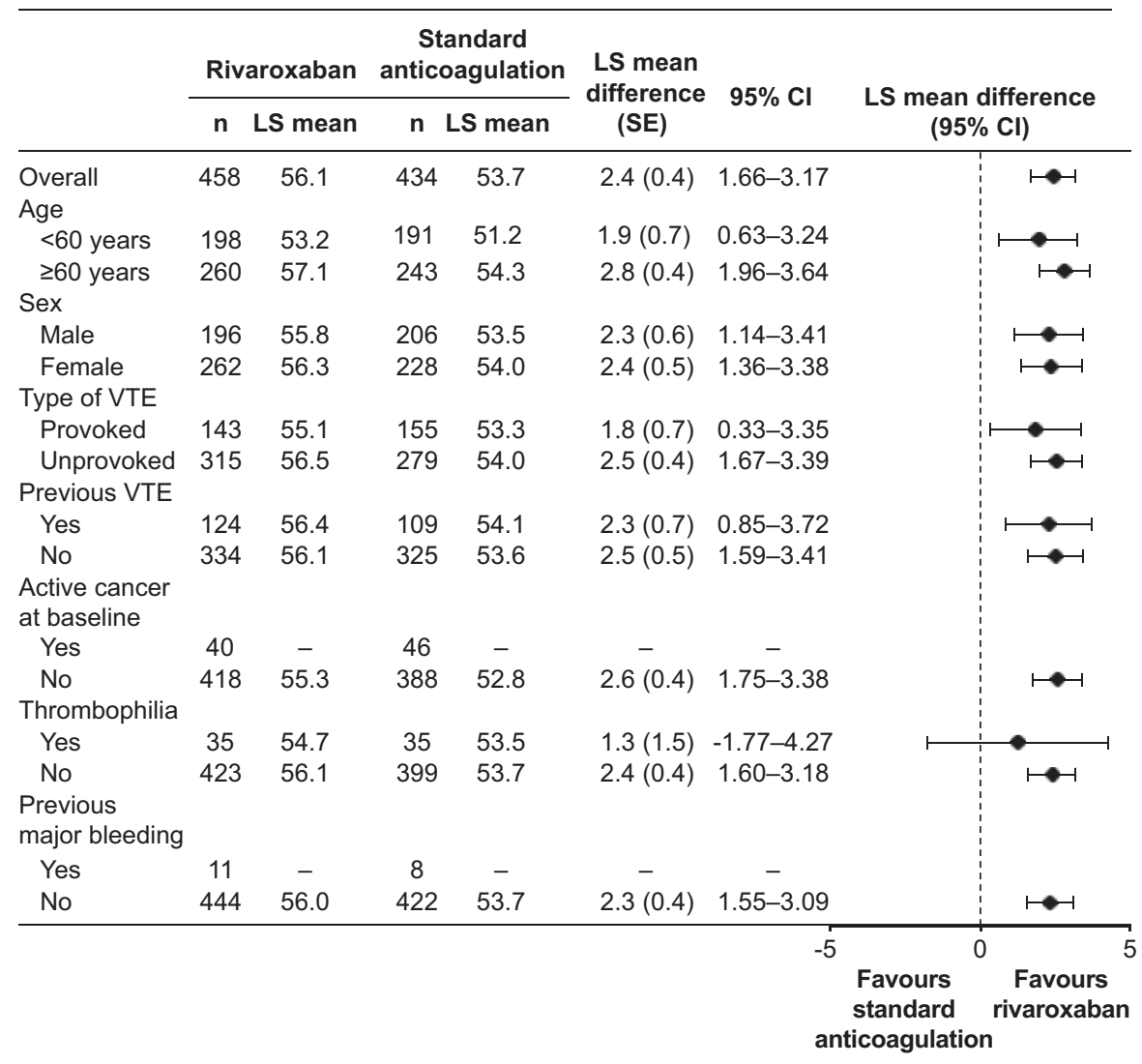

Fig. 1 ACTS Burdens score least squares mean differences by subgroup (PMS-ACTS analysis set). Subgroups with missing values had too few patients to enable LS mean difference to be calculated. ACTS, Anti-Clot Treatment Scale; Cl, confidence interval; LS, least squares; PMS, propensity score-matched set; SE, standard error; VTE, venous thromboembolism.

several advantages: although generic measures allow comparisons of outcomes across different study populations, thus enhancing the generalizability of findings, specific measures may be more sensitive for the detection and quantification of small changes that may be important to physicians or patients. $^{17}$

Another limitation of the study was that the patient population was constrained by the availability of ACTS values; the ASAF-ACTS contained only $38 \%$ of the ASAF patients resulting in 1,726 patients from eight countries (Canada, Denmark, England, France, Germany, Italy, Spain, and Sweden); therefore, caution is advised when extrapolating these results to the wider patient population.

In conclusion, despite the limitations described earlier, this substudy confirms the findings from the EINSTEIN phase III studies that the patient experience is less

Table 4 ACTS Benefits scores by visit (PMS-ACTS set)

\begin{tabular}{|l|l|l|l|l|}
\hline Visit & $\begin{array}{l}\text { Rivaroxaban/standard } \\
\text { anticoagulation } \\
\text { patients }(\mathbf{n} / \mathbf{n})^{\mathrm{a}}\end{array}$ & $\begin{array}{l}\text { Rivaroxaban/standard } \\
\text { anticoagulation } \\
\text { scores (LS mean/ } \\
\text { LS mean) }\end{array}$ & $\begin{array}{l}\text { LS mean difference } \\
\text { (SE) }\end{array}$ & $p$-Value \\
\hline Overall & $450 / 430$ & $12.1 / 11.9$ & $0.2(0.2)$ & 0.2 \\
\hline 1 & $326 / 286$ & $11.6 / 11.6$ & $-0.1(0.2)$ & 0.8 \\
\hline 2 & $298 / 277$ & $12.0 / 11.4$ & $0.5(0.2)$ & 0.01 \\
\hline 3 & $177 / 159$ & $12.1 / 11.9$ & $0.2(0.3)$ & 0.4 \\
\hline 4 & $68 / 75$ & $12.2 / 12.1$ & $0.1(0.4)$ & 0.8 \\
\hline 5 & $67 / 77$ & $12.3 / 12.2$ & $0.1(0.3)$ & 0.8 \\
\hline
\end{tabular}

Abbreviations: ACTS, Anti-Clot Treatment Scale; LS, least squares; PMS, propensity score matched set; SE, standard error.

${ }^{a}$ ACTS Benefits scores showed an odds ratio of 0.91 (95\% Cl: 0.84-0.98) for treatment discontinuation in the PMS-ACTS analysis set; therefore, the lower the ACTS Benefits score was, the more patients tended to discontinue from the study. 
Table 5 ACTS Benefits scores by visit (ASAF-ACTS set)

\begin{tabular}{|l|l|l|l|l|}
\hline Visit & $\begin{array}{l}\text { Rivaroxaban/standard } \\
\text { anticoagulation } \\
\text { patients }(\boldsymbol{n} / \boldsymbol{n})\end{array}$ & $\begin{array}{l}\text { Rivaroxaban/standard } \\
\text { anticoagulation } \\
\text { scores (LS mean/ } \\
\text { LS mean) }\end{array}$ & $\begin{array}{l}\text { LS mean difference } \\
\text { (SE) }\end{array}$ & $p$-Value \\
\hline Overall & $989 / 712$ & $11.9 / 11.8$ & $0.2(0.1)$ & 0.4 \\
\hline 1 & $724 / 465$ & $11.8 / 11.6$ & $0.2(0.2)$ & 0.5 \\
\hline 2 & $639 / 447$ & $11.8 / 11.6$ & $0.1(0.2)$ & 0.3 \\
\hline 3 & $365 / 244$ & $11.9 / 11.8$ & $0.2(0.3)$ & 0.6 \\
\hline 4 & $165 / 119$ & $12.1 / 11.9$ & $0.2(0.4)$ & 0.7 \\
\hline 5 & $152 / 114$ & $12.2 / 11.9$ & $<0.1(0.4)$ & 0.5 \\
\hline
\end{tabular}

Abbreviations: ACTS, Anti-Clot Treatment Scale; ASAF, adjusted safety analysis; LS, least squares; SE, standard error.

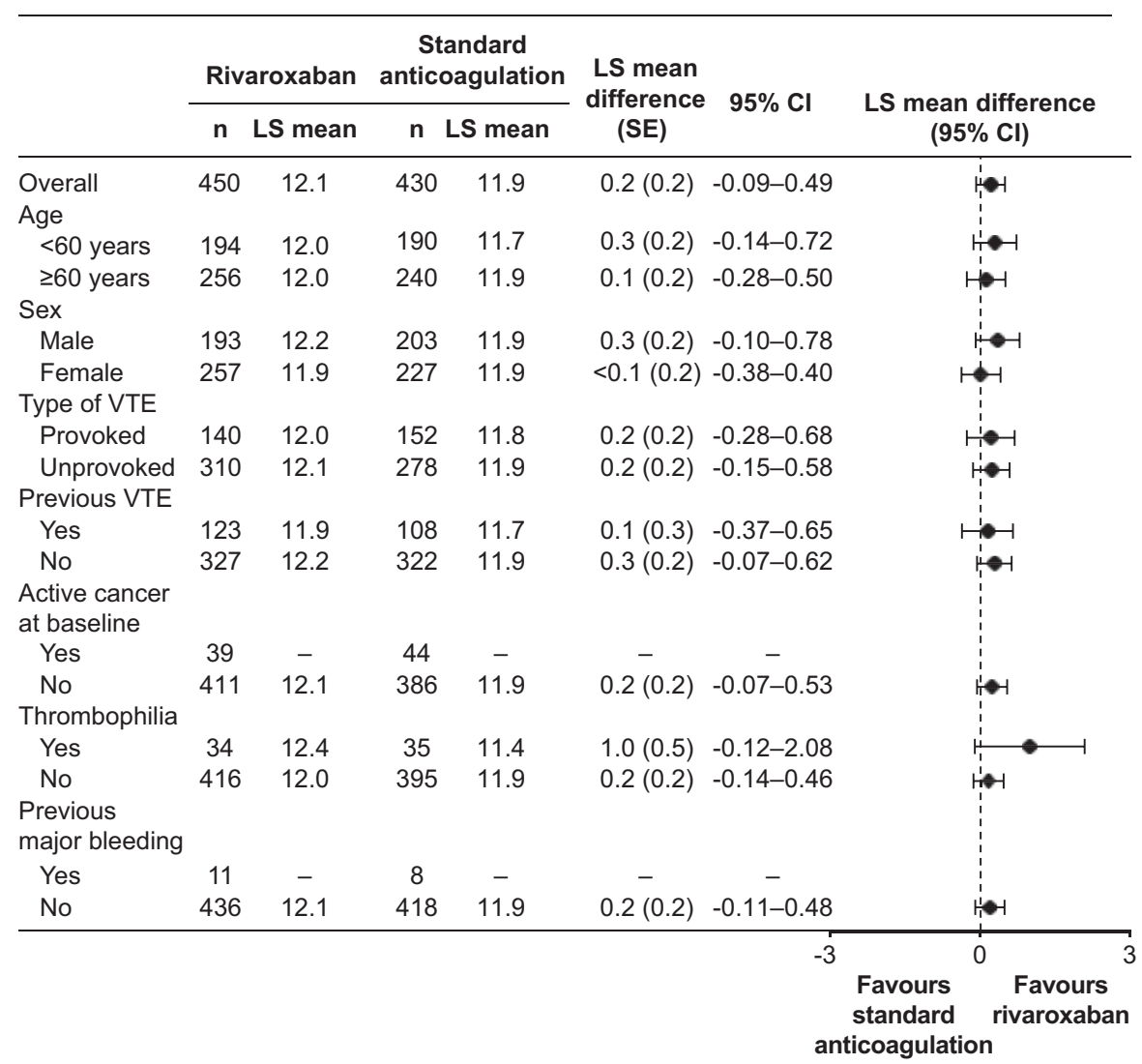

Fig. 2 ACTS Benefits score least squares mean differences by subgroup (PMS-ACTS analysis set). Subgroups with missing values had too few patients to enable LS mean difference to be calculated. ACTS, Anti-Clot Treatment Scale; CI, confidence interval; LS, least squares; PMS, propensity score-matched set; SE, standard error; VTE, venous thromboembolism.

burdensome with rivaroxaban treatment than with standard anticoagulation. This finding was expected based on the characteristics of the two therapies. The differences in ACTS Benefits scores were numerically in favor of rivaroxaban versus standard anticoagulation treatment in XALIA, although this did not reach statistical significance. These findings may potentially impact on factors such as treatment adherence and persistence and could, therefore, positively impact patients who have a long-term requirement for anticoagulation treatment.

\section{Trial Registration Number NCT01619007.}

\section{Authors' Contributions}

S.C. created the initial draft of this report. K.S., L.M., K.F., A. G.G.T., S.H., R.K., J.S., M.v.E., and W.A. provided input into the content and direction of the manuscript. M.G. performed the statistical analyses and E.Z. did the propensity score design. D.M. was responsible for data management. All authors had full access to the source data and 
participated in writing and reviewing the report and accept full responsibility for its overall content.

Conflicts of Interest

S.C. has received consultancy fees from Bayer. M.G., K.S., K.F., D.M., J.S., and M.v.E. are employees of Bayer AG. L.M. has received consultancy fees from Bayer and Daiichi Sankyo, and research support from Boehringer Ingelheim, JanssenCilag Ltd, and Pfizer Inc. A.G.G.T. has received speaker's honoraria and consultancy fees from, and participated in scientific advisory boards for, Bayer and Janssen Research \& Development, LLC. S. H. has received consultancy fees from Aspen Pharmacare, Bayer, Bristol-Myers Squibb, Daiichi Sankyo, Pfizer Inc., and Sanofi SA. R.K. has received consultancy fees from Bayer, Berlin-Chemie Menarini, Daiichi Sankyo, Lundbeck Ltd, and Servier Laboratories Ltd, and speaker's honoraria from Bayer, Bristol-Myers Squibb, and Daiichi Sankyo. E.Z. has received consultancy fees from Bayer. W.A. has received speaker's honoraria from, and participated in scientific advisory boards for, Boehringer Ingelheim, Bayer, Bristol-Myers Squibb/Pfizer, and Daiichi Sankyo, and has received research support from Bayer.

\section{Funding}

Editorial assistance was supported by Bayer AG and Janssen Scientific Affairs, LLC.

\section{Acknowledgments}

The information contained in this article is presented on behalf of the XALIA Investigators. We thank Robert Gillies (medical writer) of Chameleon Communications International, United Kingdom, who provided editorial assistance funded by Bayer AG and Janssen Scientific Affairs, LLC.

\section{References}

1 Mengiardi S, Tsakiris DA, Lampert ML, Hersberger KE. Drug use problems with self-injected low-molecular-weight heparins in primary care. Eur J Clin Pharmacol 2011;67(02):109-120

2 Hamilton JG. Needle phobia: a neglected diagnosis. J Fam Pract 1995;41(02):169-175

3 Erkens PM, ten Cate H, Büller HR, Prins MH. Benchmark for time in therapeutic range in venous thromboembolism: a systematic review and meta-analysis. PLoS One 2012;7(09):e42269
4 Wells PS, Holbrook AM, Crowther NR, Hirsh J. Interactions of warfarin with drugs and food. Ann Intern Med 1994;121(09): 676-683

5 Osterberg L, Blaschke T. Adherence to medication. N Engl J Med 2005;353(05):487-497

6 Barbosa CD, Balp MM, Kulich K, Germain N, Rofail D. A literature review to explore the link between treatment satisfaction and adherence, compliance, and persistence. Patient Prefer Adherence 2012;6:39-48

7 Bauersachs R, Berkowitz SD, Brenner B, et al; EINSTEIN Investigators. Oral rivaroxaban for symptomatic venous thromboembolism. N Engl J Med 2010;363(26):2499-2510

8 Büller HR, Prins MH, Lensin AW, et al; EINSTEIN-PE Investigators. Oral rivaroxaban for the treatment of symptomatic pulmonary embolism. N Engl J Med 2012;366(14):1287-1297

9 Bamber L, Wang MY, Prins MH, et al. Patient-reported treatment satisfaction with oral rivaroxaban versus standard therapy in the treatment of acute symptomatic deep-vein thrombosis. Thromb Haemost 2013;110(04):732-741

10 Prins MH, Bamber L, Cano SJ, et al. Patient-reported treatment satisfaction with oral rivaroxaban versus standard therapy in the treatment of pulmonary embolism; results from the EINSTEIN PE trial. Thromb Res 2015;135(02):281-288

11 Ageno W, Mantovani LG, Haas S, et al. Safety and effectiveness of oral rivaroxaban versus standard anticoagulation for the treatment of symptomatic deep-vein thrombosis (XALIA): an international, prospective, non-interventional study. Lancet Haematol 2016;3(01):e12-e21

12 Dahabreh IJ, Sheldrick RC, Paulus JK, et al. Do observational studies using propensity score methods agree with randomized trials? A systematic comparison of studies on acute coronary syndromes. Eur Heart J 2012;33(15):1893-1901

13 Collins GS, Le Manach Y. Comparing treatment effects between propensity scores and randomized controlled trials: improving conduct and reporting. Eur Heart J 2012;33(15):1867-1869

14 Parsons LS. Performing a $1: \mathrm{N}$ case-control match on propensity score. SAS Users Group International. Montreal, Canada, May 912, 2004, Poster 165-29. Available at: http://www2.sas.com/ proceedings/sugi29/165-29.pdf. Accessed April 24, 2017

15 Cano SJ, Lamping DL, Bamber L, Smith S. The Anti-Clot Treatment Scale (ACTS) in clinical trials: cross-cultural validation in venous thromboembolism patients. Health Qual Life Outcomes 2012; 10:120

16 Atkinson MJ, Kumar R, Cappelleri JC, Hass SL. Hierarchical construct validity of the treatment satisfaction questionnaire for medication (TSQM version II) among outpatient pharmacy consumers. Value Health 2005;8(Suppl 1):S9-S24

17 Patrick DL, Deyo RA. Generic and disease-specific measures in assessing health status and quality of life. Med Care 1989;27(3, Suppl):S217-S232 